\title{
Modeling a space weather event from the Sun to the Earth: CME generation and interplanetary propagation
}

\author{
Ward B. Manchester IV, Tamas I. Gombosi, Ilia Roussev, Aaron Ridley, \\ Darren L. De Zeeuw, I. V. Sokolov, and Kenneth G. Powell \\ Center for Space Environment Modeling, University of Michigan, Ann Arbor, Michigan, USA
}

Gábor Tóth

Center for Space Environment Modeling, University of Michigan, Ann Arbor, Michigan, USA

Department of Atomic Physics, Eotvos University, Budapest, Hungary

Received 18 July 2003; revised 13 November 2003; accepted 24 November 2003; published 10 February 2004.

[1] We present a three-dimensional (3-D) numerical ideal magnetohydrodynamics (MHD) model describing the time-dependent expulsion of a coronal mass ejection (CME) from the solar corona propagating to 1 astronomical unit (AU). The simulations are performed using the Block Adaptive Tree Solar-Wind Roe Upwind Scheme (BATS-R-US) code. We begin by developing a global steady-state model of the corona that possesses high-latitude coronal holes and a helmet streamer structure with a current sheet at the equator. The Archimedean spiral topology of the interplanetary magnetic field is reproduced along with fast and slow speed solar wind. Within this model system, we drive a CME to erupt by the introduction of a Gibson-Low magnetic flux rope that is anchored at both ends in the photosphere and embedded in the helmet streamer in an initial state of force imbalance. The flux rope rapidly expands and is ejected from the corona with maximum speeds in excess of $1000 \mathrm{~km} / \mathrm{s}$. Physics-based adaptive mesh refinement (AMR) allows us to capture the structure of the CME focused on a particular Sun-Earth line with high spatial resolution given to the bow shock ahead of the flux rope as well as to the current sheet behind. The CME produces a large magnetic cloud at $1 \mathrm{AU}\left(>100 R_{\odot}\right)$ in which Bz undergoes a full rotation from north to south with an amplitude of $20 \mathrm{nT}$. In a companion paper, we find that the $\mathrm{CME}$ is very effective in generating strong geomagnetic activity at the Earth in two ways. First, through the strong sustained southward $\mathrm{Bz}$ (lasting more than 10 hours) and, second, by a pressure increase associated with the CMEdriven shock that compresses the magnetosphere. INDEX TERMS: 7513 Solar Physics, Astrophysics, and Astronomy: Coronal mass ejections; 7524 Solar Physics, Astrophysics, and Astronomy: Magnetic fields; 2784 Magnetospheric Physics: Solar wind/magnetosphere interactions; 2139 Interplanetary Physics: Interplanetary shocks; 7851 Space Plasma Physics: Shock waves; KEYWORDS: coronal mass ejection, magnetohydrodynamics, space weather

Citation: Manchester, W. B., IV, T. I. Gombosi, I. Roussev, A. Ridley, D. L. De Zeeuw, I. V. Sokolov, K. G. Powell, and G. Tóth (2004), Modeling a space weather event from the Sun to the Earth: CME generation and interplanetary propagation, J. Geophys. Res., 109, A02107, doi:10.1029/2003JA010150.

\section{Introduction}

[2] Coronal mass ejections (CMEs) have traditionally been defined as large-scale expulsions of plasma from the corona seen as bright arcs in coronagraphs that record Thomson scattered light. These events are the most stunning activity of the solar corona in which typically $10^{15}-10^{16} \mathrm{~g}$ of plasma is hurled into interplanetary space with a kinetic energy the order of $10^{31-32}$ ergs. It is also believed that these eruptions are associated with large-scale reconfigurations of the coronal magnetic field that contribute to the magnetic polarity reversal over the solar cycle [Low, 2001].

Copyright 2004 by the American Geophysical Union. 0148-0227/04/2003JA010150
Because of their energetics and global scale, CMEs are of intense theoretical interest, but CMEs are also of equally great practical interest due to the large disturbances they produce in the solar wind that are the primary cause of nonrecurrent geomagnetic storms [Gosling, 1993]. CMEs typically drive such storms in two ways. First, by way of a prolonged southward interplanetary magnetic field (IMF) at 1 astronomical unit (AU) that allows a strong coupling between the solar wind and the magnetosphere [Dungey, 1961]. This geoeffective magnetic field associated with the CME may result from the distortion and amplification of the ambient IMF, or it may be the manifestation of coronal magnetic ejecta reaching the Earth in the form of a magnetic cloud. Magnetic clouds have the properties of a strong 
magnetic field (relative to the ambient IMF), smooth field rotation and low temperature [Burlaga et al., 1981]. These structures are interpreted as magnetic flux ropes ejected from the corona into the solar wind during CMEs [Burlaga et al., 1982]. In addition to magnetic driving, goemagnetic storms may be caused by a pressure increase associated with CME-driven shocks that can significantly compress the magnetosphere and be as geoeffective as strong sustained southward IMF.

[3] The problem of CME propagation from the corona to $1 \mathrm{AU}$ is one component of space weather that concerns the physical conditions and dynamics of the coupled system comprising the Sun, solar wind, magnetosphere, ionosphere and thermosphere. Solutions to the equations of magnetohydrodyamics (MHD) are the only self-consistent mathematical description of the Sun-Earth space environment that can span the necessary range of length-scales to provide a truly global model of the system. Although MHD offers only a low order approximation of the state of the plasma, it does capture the bulk transport of the plasma and magnetic flux that are essential to space weather. An example of the early MHD treatment of CME transport is provided by Chen and Garren [1993], who developed a model describing the propagation of flux ropes to $1 \mathrm{AU}$ that included the Lorentz force, pressure gradients and drag forces in an integrated dynamical equation. Kumar and Rust [1996] obtained scaling laws based on magnetic flux, helicity and mass conservation to relate magnetic clouds at $1 \mathrm{AU}$ to flux ropes initially located in the corona. The first numerical simulations of heliospheric disturbances such as CMEs were modeled by the injection of dense plasma in an inner boundary placed beyond the critical point $r>20 R_{\odot}$ [e.g., Odstrcil and Pizzo, 1999]. These models give physical insight into how a large disturbance travels and interacts with the large-scale solar wind but rely on ad hoc inputs to the solar wind without considering evolution of such a structure from the corona. Only recently has the propagation of a CME from the inner corona to $1 \mathrm{AU}$ been numerically modeled [e.g., Usmanov and Dryer, 1995], [Wu et al., 1999; Groth et al., 2000; Riley et al., 2002; Odstrcil et al., 2002]. Of these models, those of Usmanov and Dryer and Groth et al. are three-dimensional, the others being two-dimensional.

[4] Coronagraph observations have shown that the majority of CMEs originate from the disruption of closed magnetic structures known as helmet streamers [Hundhausen, 1987, 1993]. It is now believed that the breakup of helmet streamers is the possible result of a loss of equilibrium following a slow, nearly quasi-static evolution [Low, 1983]. Several mechanisms have been invoked to initiate CMEs. In the case of flux rope-driven events, the injection of azimuthal magnetic flux has been used to drive eruptions as done by Chen [1996] and Wu et al. [1999]. Localized magnetic reconnection has also been introduced to sever field lines and allow a portion of the system (typically a flux rope) to expand upward [e.g., Forbes and Priest, 1995; Lin and Forbes, 2000; Chen and Shibata, 2000]. A combination of system driving and magnetic reconnection has been used to model the initiation of CMEs. In the most common example, two-dimensional (2-D) magnetic arcades are made to approach an open state by way of prescribed footpoint motions that shear the magnetic field. Reconnection, when applied within the shearing arcade results in eruption [Steinolfson, 1991; Choe and Lee, 1996; Linker and Mikice, 1995]. Antiochos et al. [1999] have produced a new variation of this model, employing a quadrupole field that allows magnetic reconnection to occur higher in the corona, which removes the unsheared field above a low-lying sheared core.

[5] The approach we take to modeling a CME propagation is to start with a system that is initially out of equilibrium and simulate its subsequent time evolution. We begin by numerically forming a steady-state corona along with a bi-modal solar wind based on the model of Groth et al. [2000]. The model coronal magnetic field is representative of a solar minimum configuration with open polar field lines and low latitude closed field lines forming a streamer belt. Having attained this steady state, we then place a three-dimensional (3-D) magnetic flux rope within the streamer belt, which is tied at both ends to the inner boundary. The flux rope we use is taken from a family of 3-D analytical solutions derived by Gibson and Low [1998], hereafter GL. The combined models of GL and Groth et al. produce a fully 3-D flux rope-driven model of a CME originating from the coronal streamer belt and propagating in a realistic bi-modal solar wind to $1 \mathrm{AU}$. The application of a flux rope to the streamer belt follows from the belief that CMEs originate from flux ropes supporting prominences within coronal streamers. In our model, we insert a flux rope out of equilibrium in the streamer belt that rapidly expands, driving a strong shock ahead of it as it is expelled from the corona along with large amounts of plasma mimicking a CME. Plasma parameters from this model at 1 AU are then used to drive a global magnetosphereionosphere model of Ridley et al. [2001]. Together these models illustrate our magnetohydrodynamic (MHD) based space weather model of the entire solar-terrestrial environment that is able to treat major geomagnetic storms. This coupled model is the basis for the development of our more sophisticated space weather prediction tools.

[6] The organization of the paper is as follows. We give a brief description of the conservative form of the equations of MHD and the scheme used to solve them in section 2 . Details of the steady-state corona and solar wind are given in section 3 , while an outline of the Gibson-Low flux rope is given in section 4. Results of the numerical simulation of the flux rope-driven $\mathrm{CME}$ are given in section 5, which includes a discussion of the morphology of the CME, its propagation to $1 \mathrm{AU}$ and plasma properties and geoeffectiveness. Finally, in section 6, we discuss the simulation results and demonstrate our 3-D flux rope-driven CME model as a component of a global space weather model.

\section{Governing Equations of the MHD Model}

[7] In our model of the corona, solar wind, and CME, we assume that the systems are composed of magnetized plasma that behaves as an ideal gas with a polytropic index, $\gamma=\frac{5}{3}$. The plasma is assumed to have infinite electrical conductivity so that the magnetic field is "frozen" into the plasma. Gravitational forces on the plasma are included, but only forces due to the Sun; there is no self gravitation of the plasma. Finally, volumetric heating of the plasma of a chosen form is assumed to occur in the corona. With these 
assumptions, the evolution of the system may be modeled by the ideal MHD equations written in conservative form:

$$
\begin{gathered}
\frac{\partial \rho}{\partial t}+\nabla \cdot(\rho \mathbf{u})=0 \\
\frac{\partial(\rho \mathbf{u})}{\partial t}+\nabla \cdot\left[\rho \mathbf{u} \mathbf{u}+\left(p+\frac{B^{2}}{8 \pi}\right) I-\frac{\mathbf{B} \mathbf{B}}{4 \pi}\right]=\rho \mathbf{g} \\
\frac{\partial \mathbf{B}}{\partial t}+\nabla \cdot(\mathbf{u} \mathbf{B}-\mathbf{B} \mathbf{u})=0 \\
\frac{\partial \varepsilon}{\partial t}+\nabla \cdot\left[\mathbf{u}\left(\varepsilon+p+\frac{B^{2}}{8 \pi}\right)-\frac{(\mathbf{u} \cdot \mathbf{B}) \mathbf{B}}{4 \pi}\right] \\
=\rho \mathbf{g} \cdot \mathbf{u}+(\gamma-1) Q
\end{gathered}
$$

where $\rho$ is the plasma mass density, $\mathbf{u}$ the plasma velocity, $\mathbf{B}$ the magnetic field, and $p$ is the plasma pressure (sum of the electron and ion pressures). The volumetric heating term, $Q$, parameterizes the effects of coronal heating as well as heat conduction and radiation transfer (see section 3 ). The gravitational acceleration is defined as $\mathbf{g}=-g(\mathbf{r} / r)\left(R_{\odot}\right)^{2}$ where $R_{\odot}$ is the solar radius and $g$ is the gravitational acceleration at the solar surface. The total energy density, $\varepsilon$, is given by

$$
\varepsilon=\frac{\rho u^{2}}{2}+\frac{p}{\gamma-1}+\frac{B^{2}}{8 \pi}
$$

where gravity is omitted from the total since it is treated as a momentum source term. This system of eight equations details the transport of mass, momentum and energy with three equations describing the evolution of the magnetic field given by Faraday's Law assuming infinite electrical conductivity. These equations are then put in dimensionless form, using values of the density and ion-acoustic wave speed from a suitable part of the physical domain (in this case the low corona) in addition to a reference length scale (in this case the solar radius). The dimensionless equations are then solved, using the block-adaptive tree solar-wind Roe-type upwind scheme (BATS-R-US) code [Powell et al., 1999; Groth et al., 2000]. This code is designed to run efficiently on massively parallel computers and solves the equations of MHD with the use of block adaptive mesh refinement (AMR). This feature of the grid allows for orders of magnitude variation in numerical resolution within the computational domain. Such resolution is necessary for global coronal models, which strive to resolve structures such as shocks and electric current sheets in a domain that extends to many solar radii.

\section{Steady-State Model of the Solar Wind}

[8] In order to simulate the time dependent behavior of a CME propagating from the low corona through the solar wind, a representative MHD model of the steadystate background solar wind is required. With such a model, the evolution of a CME is then formulated as a propagation problem with the initial condition of the corona and solar wind specified by the steady-state solution.

[9] In this section, we describe our steady-state model of the corona and solar wind that is designed to reproduce conditions near solar minimum. The essential features of this model are as follows: (1) open magnetic field lines forming coronal holes at high latitude; (2) closed magnetic field lines forming a streamer belt near the Sun at low latitudes; (3) the bi-modal nature of the solar wind is reproduced with fast wind over the poles and slow wind at low latitudes. A thin current sheet forms at the tip of the streamer belt and separates opposite directed magnetic flux originating from the two poles. The model is simplified by alignment of the magnetic axis with the $z$ axis so the solution is axisymmetric. Also, solar rotation is included since the domain extends to more than 300 solar radii at which distance the azimuthal component of the Parker [1963] spiral is significant. While the magnetic field structure of this steady-state solution is simple, future models will eventually incorporate synoptic magnetogram observations for more realistic magnetic field configurations.

[10] The corona is composed of high temperature $(T>$ $10^{6} \mathrm{~K}$ ) low density $\left(\rho \approx 10^{-16} \mathrm{gm} \mathrm{cm}^{-3}\right.$ ) plasma composed primarily of ionized hydrogen. The temperature and pressure of the corona are such that it can not be held in equilibrium by solar gravity or the pressure of the interstellar medium. Consequently, it expands outward at supersonic speeds and in doing so forms the solar wind [Parker, 1963]. The fast solar wind originates from coronal holes and is possibly driven to high velocity by additional heating that occurs close to the Sun [cf. Axford and McKenzie, 1996]. It is also thought that momentum deposited by Alfvén waves may accelerate this component of the wind [cf. Hollweg et al., 1982; Usmanov et al., 2000] that lies at heliolatitudes $>22^{\circ}$ at solar minimum. In our model, we find that the fast wind has a velocity of $750 \mathrm{~km} \mathrm{~s}^{-1}$ at distances greater than 15 solar radii. Beyond this distance, the plasma has cooled to such an extent that there is little acceleration of the wind. The slow wind by contrast is confined close to the global heliospheric current sheet, which lies near the equator at solar minimum. This component of the wind is observed to be highly variable with speeds that lie in the slow-tomedium range between 340 to $700 \mathrm{~km} \mathrm{~s}^{-1}$. The source of the slow wind may be highly expanded plasma traveling down magnetic flux tubes that originate near coronal hole boundaries. It has also been suggested that opening of closed flux tubes by interchange reconnection with open flux may release plasma to form the slow solar wind [Fisk et al., 1999].

[11] The steady-state model of the corona and solar wind described here was first developed by Groth et al. [2000]. However, we have made significant modifications to the original steady-state model to suit our purposes. First, our simulation is performed in the inertial frame rather than the corotating frame. Second, the magnetic axis is aligned with the $z$ axis in our model rather than having a tilted intrinsic magnetic field topology. Third, we have slightly modified the inner boundary condition to allow for the flux rope to be line-tied to the coronal surface. Finally, our model of the solar wind extends to $336 R_{\odot}$ compared to $224 R_{\odot}$ of the 
original model with a more highly refined numerical grid. In all other ways, our steady-state model is identical to Groth et al. [2000], that we briefly outline here.

[12] The steady-state numerical model is made with the assumption that the base of the corona is at the inner boundary and acts as a reservoir of hot plasma with an embedded magnetic field. The temperature of that plasma is taken to be $2.85 \times 10^{6} \mathrm{~K}$ with a plasma density of $\rho=2.5 \times$ $10^{-16} \mathrm{gm} \mathrm{cm}^{-3}$. The intrinsic magnetic field at the solar surface, $\mathbf{B}_{0}$, may be written as a multipole expansion of the following form:

$$
B_{0 k}=3 \frac{M_{i} r_{i}}{r^{4}} \frac{r_{k}}{r}-\frac{M_{k}}{r^{3}}+7 \frac{Q_{i j l} r_{i} r_{j} r_{l}}{r^{8}} \frac{r_{k}}{r}-3 \frac{Q_{i j k} r_{i} r_{j}}{r^{7}}
$$

where $M_{i}(i=1,2,3)$ are the components of the dipole moment vector and $Q_{i j k}$ are the octupole moment tensor components with the octupole aligned with the $z$ axis (there is no quadrupole moment in this model). Repeated indices are summed over from 1 to 3 where $r_{1}=x, r_{2}=y$, and $r_{3}=z$. We have taken the dipole aligned with the $z$ axis so that $M_{x}=$ $M_{y}=0$ and $M_{z}=b_{0}$. The dipole and octupole moments are chosen such that the maximum field strength at the poles is 8.4 Gauss and 2.2 Gauss at the equator.

[13] Volumetric heating of the model corona is introduced in a way that attempts to mimic the effects of energy absorption above the transition region, thermal conduction and radiative losses as well as satisfying known constraints of coronal heating. The heating function has the following form,

$$
Q=\rho q_{0}\left(T_{0}-\gamma \frac{p}{\rho}\right) \exp \left[-\frac{\left(r-R_{\odot}\right)^{2}}{\sigma_{0}^{2}}\right]
$$

where the target temperature, $T_{0}$, is $5.0 \times 10^{6} \mathrm{~K}$ poleward from a critical angle $\theta_{0}(r)$, which defines the coronal hole boundary, while $T_{0}=2.85 \times 10^{6} \mathrm{~K}$ equatorward of $\theta_{0}(r)$ in the streamer belt. The heating function is defined with $q_{0}=$ $10^{6} \mathrm{ergs} \mathrm{g}^{-1} \mathrm{~s}^{-1} \mathrm{~K}^{-1}$, where $R_{\odot}$ is equal to the solar radius and $r$ is the heliocentric radius. The function $\theta_{0}(r)$ is defined as follows: for the region $R_{\odot}<r<7 R_{\odot}$,

$$
\sin ^{2}\left(\theta_{o}\right)=\sin ^{2}\left(17.5^{\circ}\right)+\frac{\left(1-\sin ^{2}\left(17.5^{\circ}\right)\right)(r-1)}{8}
$$

which is equal to $17.5^{\circ}$ at the solar surface and increases to a value of $\theta_{o}=61.5^{\circ}$ at $r=7 R_{\odot}$. Beyond this radius, $\theta_{o}$ increases more slowly as

$$
\sin ^{2}\left(\theta_{o}\right)=\sin ^{2}\left(61.5^{\circ}\right)+\frac{\left(1-\sin ^{2}\left(61.5^{\circ}\right)\right)(r-7)}{40}
$$

reaching a value of $\theta_{o}=90^{\circ}$ at $r=47 R_{\odot}$, which is then held fixed at $90^{\circ}$ for $r>47 R_{\odot}$. The heating scale height function is kept constant in the streamer belt with $\sigma_{0}(r, \theta)=4.5$, and increases inside the coronal hole as

$$
\sigma_{0}(r, \theta)=4.5\left(2-\frac{\sin ^{2}(\theta)}{\sin ^{2}\left(\theta_{o}\right)}\right)
$$

which gives a scale height for the volumetric heating that varies from $4.5 R_{\odot}$ near to equator to $9 R_{\odot}$ at the poles. It is important to mention that this heating function was designed with several free parameters with the desire of reproducing observed features of the fast and slow solar wind.

\subsection{Computational Mesh}

[14] The computational domain for the simulation extends from $-192 R_{\odot}<x<192 R_{\odot},-48 R_{\odot}<y<$ $336 R_{\odot}$, and $-192 R_{\odot}<z<192 R_{\odot}$ with the Sun placed at the origin with the magnetic axis aligned with the $z$ axis. The system is initially resolved with 22,772 self-similar $6 \times$ $6 \times 6$ blocks containing a total of 4.9 million cells. The blocks are distributed in size over eight levels of refinement with each subsequent level of refinement using cells half the size of the previous level (in each dimension). In this case, cells range in size from $1 / 32 R_{\odot}$ to $4 R_{\odot}$ and are spatially positioned to highly resolve the central body and the flux rope as well as the heliospheric current sheet. The grid is refined every 100 iterations during the first 10 hours after CME initiation with the frequency reduced to every 200 iterations thereafter. AMR criteria are chosen so that blocks close to the Sun-Earth line (in this case, near the $y$ axis) with large time variations in density are preferentially refined. The high resolution mesh tracks the shock directly in front of the flux rope and trails behind to also resolve the flux rope and the field lines connecting the flux rope to the Sun. This refinement is essential to slow the rate of reconnection behind the flux rope. Both refinement and coarsening are performed to allow the total number of cells to eventually grow over 13.8 million to fill the ever expanding volume of space occupied by the CME.

\subsection{Boundary Conditions}

[15] To pose a physically meaningful system subject to the equations of ideal MHD, it is necessary to specify appropriate boundary conditions at the inner boundary (the spherical surface $r=R_{\odot}$ ) and the outer boundary (the outer surfaces of the rectangular domain). The coronal boundary conditions are a function of heliospheric latitude. In the coronal holes poleward of $\theta_{0}$, the following values are prescribed in ghost cells inside $r=R_{\odot}: \rho=2.5 \times$ $10^{-16} \mathrm{gm} \mathrm{cm}^{-3}, p=5.89 \times 10^{-2}$ dynes $\mathrm{cm}^{-2}, \mathbf{u}=0$, and $\mathbf{B}=\mathbf{B}_{0}$. These values are then allowed to interact with the solution inside our physical domain through the $r=R_{\odot}$ boundary by solving the Reimann problem at the boundary. The boundary is treated as a discontinuity in which the inside values are the prescribed boundary values and the outside values are taken from the adjacent active cell. Solution to the Reimann problem provides the fluxes of mass, energy, momentum, and magnetic field associated with the propagation of waves from the discontinuity. The fluxes at the square cell faces are then scaled according to the size and orientation of the spherical boundary passing through the cell. This approach ensures that the appropriate information from the "solar" values is propagated into the solution domain by the numerical flux function used in the scheme. These conditions set up a pressure gradient that drives plasma away from the Sun and permits plasma to pass through the boundary as the mass source for the solar wind. The magnetic field at the surface is specified by the time-independent multipole expansion for the intrinsic field given by equation (6). In the streamer belt equatorward of $\theta_{0}$, the following values are prescribed just inside $r=R_{\odot}$ : 

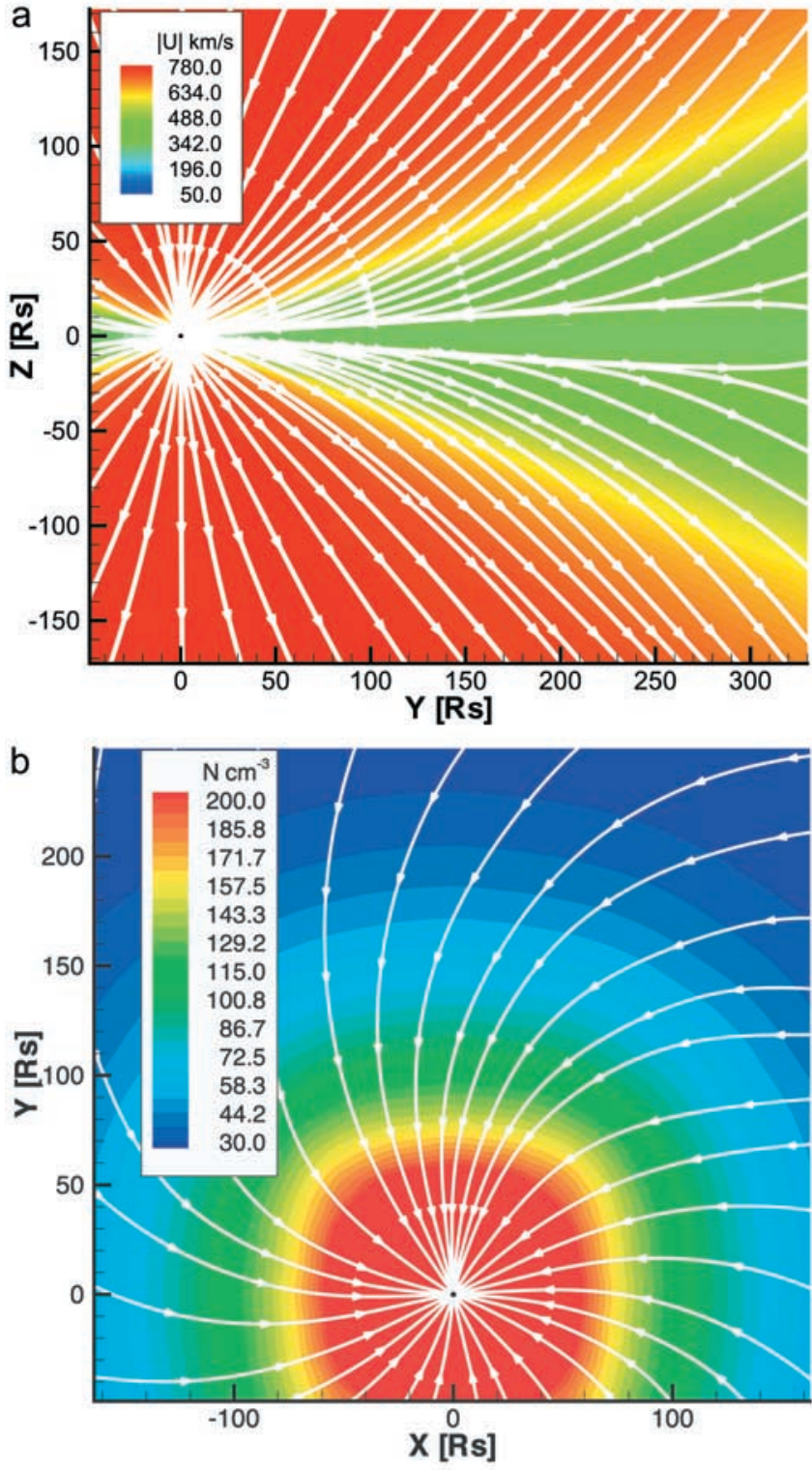

Figure 1. Magnetic field, velocity, and density for the steady-state solar wind solution. Solid white lines are magnetic "streamlines" drawn in the $y-z$ plane superimposed upon a false color image of the velocity magnitude in the top panel. Note the bi-modal nature of the solar wind. The bottom panel depicts magnetic "streamlines" showing the Parker spiral superimposed on a false color image of plasma density on the $x-y$ plane located above the current sheet at $z=25 R_{\odot}$.

$\partial \rho / \partial r=0, \partial p / \partial r=0, \mathbf{u}=-\mathbf{u}_{\text {outside }}, \mathbf{B}=\mathbf{B}_{\text {outside }}$, where the subscript outside refers to the values just outside $r=R_{\odot}$, which are computed by the flow solution scheme. These conditions strictly enforce a zero flow at the boundary, both in the radial and tangential directions, with the exception of solar rotational flow. The boundary conditions also enforce continuity of the magnetic field across $r=R_{\odot}$. This provision allows the magnetic field of the flux rope (prescribed in the streamer belt) to pass through the interface where the footpoints are effectively "line tied" to the rotating surface. At the outer boundary of the domain, the flow is super-fast. Thus all waves are exiting the domain, and no information from outside the domain propagates into the domain.

\subsection{Steady-State Solar Wind Solution}

[16] The solar wind solution is produced by the time evolution of the system subject to the described heating function, intrinsic magnetic field and boundary conditions. Local time stepping is used to speed up convergence, achieving a near steady-state solution after 146,000 iterations with AMR periodically applied to resolve the heliospheric current sheet. Figure 1 depicts two two-dimensional (2-D) cuts through the 3-D steady-state model with top and bottom panels showing a meridional slice and a slice near the equatorial plane respectively. The top false color image indicates the velocity magnitude, $|\mathbf{u}|$, of the plasma while the magnetic field is represented by solid white lines. The magnetic field remains closed at low latitude close to the Sun, forming a streamer belt. At high latitude, the magnetic field is carried out with the solar wind to achieve an open configuration. Closer to the equator, closed loops are drawn out and at a distance $\left(r>3 R_{\odot}\right)$, collapse into a field reversal layer. The resulting field configuration has a neutral line and a current sheet originating at the tip of the streamer belt similar to numerical solution originally obtained by Pneuman and Kорp [1971]. Even after 140,000 iterations, the helmet streamer is still slowly evolving as closed loops flow out with the wind and small meridional flows close to the sun move open flux closer to the equator. The relative strength of the non-radial magnetic field produced by these flows is magnified as the distance from the Sun increases. The bottom false color image shows magnetic stream lines in white formed to a Parker spiral by rotation applied to the inner boundary. The false color image shows a $1 / r^{2}$ fall off in solar wind plasma density.

[17] Inspection of the top panel of Figure 1 reveals a bimodal outflow pattern with slow wind leaving the Sun below $400 \mathrm{~km} / \mathrm{s}$ near the equator and high-speed wind above $750 \mathrm{~km} / \mathrm{s}$ found above $30^{\circ}$ latitude. The variation in solar wind speed in this model results from corona heating and flux expansion. Flux expansion is measured by a factor that is the ratio of flux tube cross sectional areas taken at two locations and normalized to a purely radial increase in surface area between the same points. At the poles, the temperature is near $5 \times 10^{6} \mathrm{~K}$ and the flux expansion factor is 2.3 , while on the lowest latitude open field line, the coronal temperature has decreased to $2.8 \times 10^{6} \mathrm{~K}$ and the flux expansion factor has increased to 5.6. The variation in wind speed and flux expansion factor is similar to the empirical model proposed by Wang and Sheeley [1994] that explains solar wind speeds as being inversely related to the expansion of contained magnetic flux tubes. However, in our case, the low-latitude expansion factors are not as large as found by Wang and Sheeley [1994] and temperature variation plays a significant role in determining wind speed.

\section{Flux Rope of Gibson and Low}

[18] To initiate a CME within this coronal model, we superimpose a 3-D magnetic flux rope and its entrained plasma into the streamer belt of our steady-state coronal model (see Figure 2). We do not change the velocity of the 


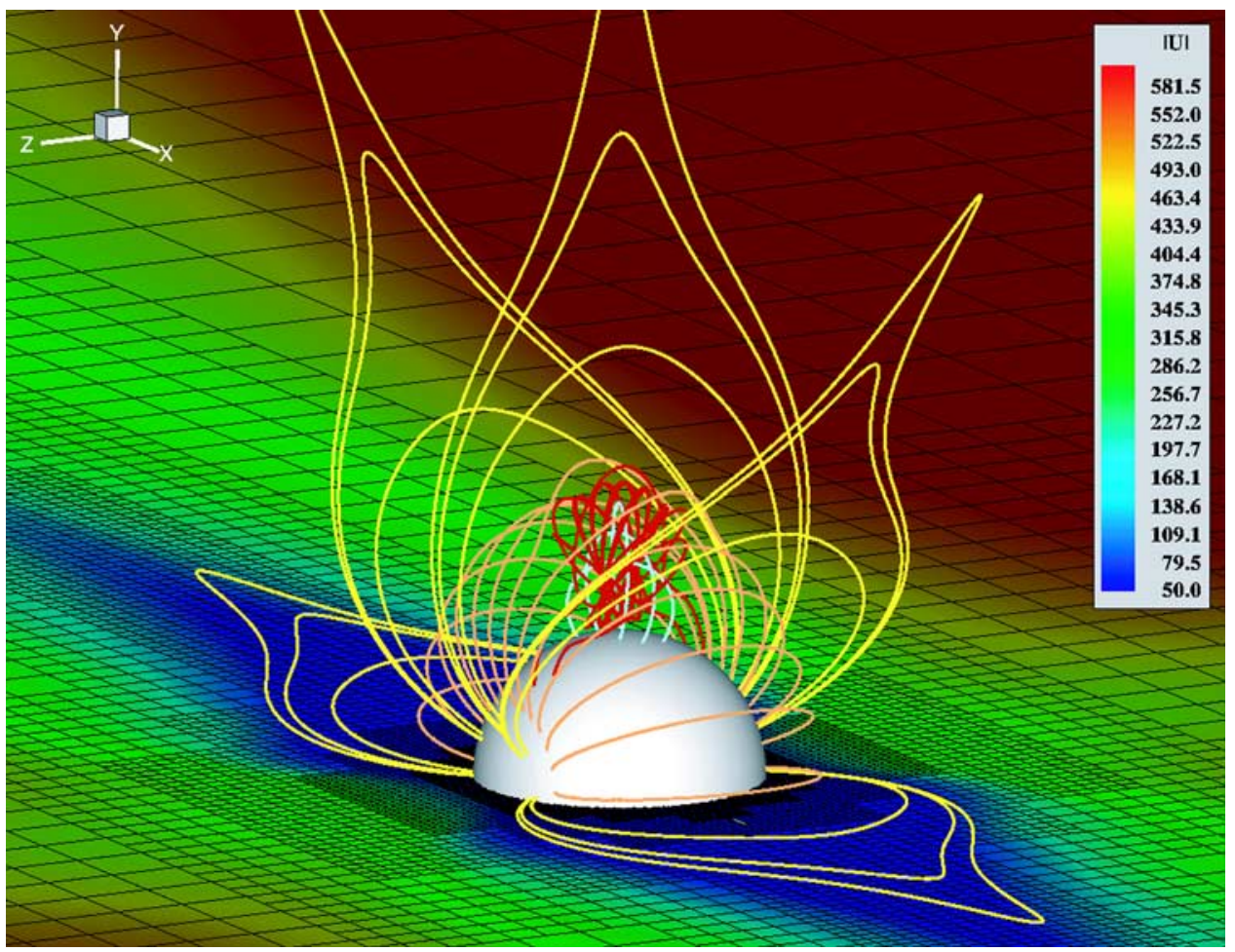

Figure 2. Depicted is a three-dimensional representation of the coronal magnetic field drawn as solid colored lines at $t=0$ hours. The flux rope is drawn with light blue and red lines showing respectively, a sheared (toroidal) core surrounded by a highly twisted sheath. Orange and yellow lines show the poloidal field of the steady-state equatorial streamer belt. On the $x-z$ plane, the computational mesh is drawn with black lines superimposed upon a false color image the velocity magnitude.

initial state to drive self-similar evolution, rather, we let the system evolve from an initial state of force imbalance. The flux rope we employ comes from the GL family of analytical solutions of the ideal MHD equations describing an idealized, self-similar expansion of a magnetized cloud resembling a CME. We briefly describe here the mathematical form of the GL solutions, while more complete derivations and descriptions of the solutions can be found in the appendix and the original paper of Gibson and Low [1998].

\subsection{Self-Similar Flux Ropes}

[19] The CME models of GL are developed by finding a 3 -D analytical solution to the equation

$$
\frac{1}{4 \pi}(\nabla \times \mathbf{B}) \times \mathbf{B}-\nabla p-\rho \mathbf{g}=0
$$

and Maxwell's equation, $\nabla \cdot \mathbf{B}=0$, that describe a global magnetostatic corona containing a magnetic flux rope in force balance with the pressure and weight of the plasma.

[20] The solution for this flux rope is derived by applying a mathematical stretching transformation $r \rightarrow r-a$ to an axisymmetric, spherical ball of twisted magnetic flux in equilibrium with plasma pressure. The transformation, performed in spherical coordinates $(r, \theta, \phi)$, draws space toward the origin while holding angular coordinates, $\theta$ and $\phi$ fixed. This mathematical procedure serves two important purposes. First, it generates a geometrically complex solution by distorting the originally spherical, axisymmetric flux rope (centered away from the heliocentric origin) into a tear- drop shape with full 3-D spatial variation. The magnetic structure, seen as a 3-D representation in Figure 2, possesses a toroidal core, shown with solid light blue lines, surrounded by flux becoming progressively more twisted closer to the flux rope surface, shown as solid red lines. The computational grid is seen in Figure 2 as black lines superimposed upon a false color image of the velocity magnitude. The second benefit of the stretching transformation is the introduction of Lorentz forces associated with the magnetic field that requires both the pressure and weight of plasma in a $1 / r^{2}$ gravitational field to be in static equilibrium. Where the magnetic field is concave away from the solar center, cold dense plasma is required to offset the upward directed magnetic tension. By contrast, where the field is convex away from the Sun, the plasma density is reduced so that buoyancy offsets the downward directed Lorentz force. The given vertical orientation of the flux rope (see Figure 2) requires a density-depleted cavity in the upper extremity of the rope, while a dense core exists in the lower portion as seen in Figure 3. This figure shows a meridional slice of the corona centered on the flux rope. Density is represented as a false color image superimposed with white lines representing the magnetic field. The density structure of the model is an attractive feature of the model in that it possesses a dense helmet streamer containing a cavity embedded with a prominence-type density enhancement. Such long-lived coronal structures are often observed to give rise to CMEs [cf. Hundhausen, 1993].

[21] With our inclusion of the GL flux rope to a numerical, steady-state model of the corona and solar wind, we 


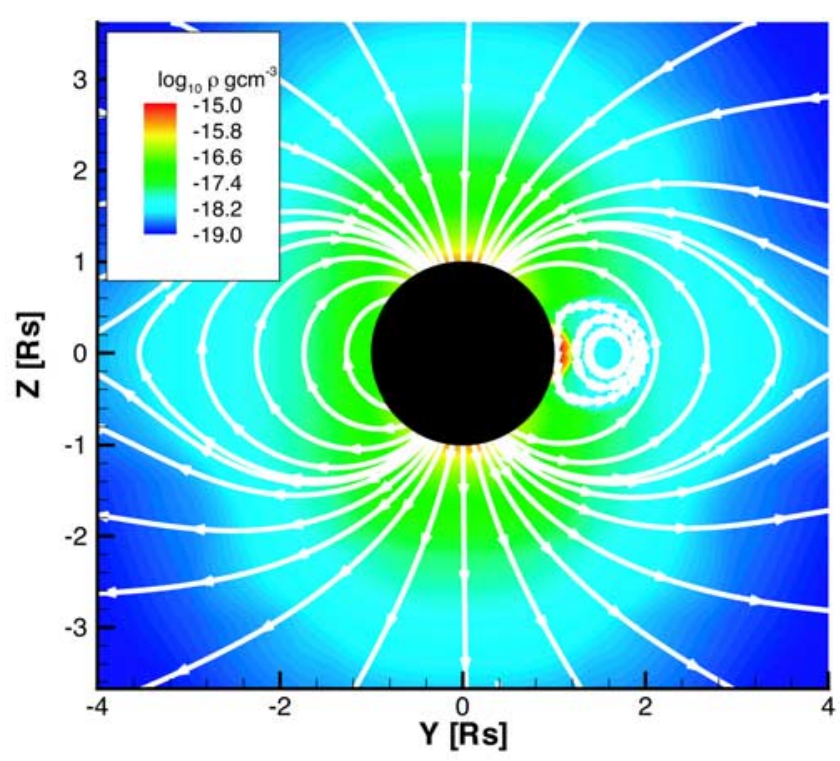

Figure 3. The initial coronal plasma density is depicted with a false color image in the $y-z$ meridional plane with the magnetic field represented by solid white "streamlines".

can allow the GL flux rope to interact self consistently with a realistic structured solar wind. We will determine to what degree a 3-D magnetic flux rope ejected from the low corona as a CME can evolve to form a magnetic cloud structure as observed at 1 AU. To begin, we do not prescribe a flow field to the flux rope and surrounding corona, but rather the CME results from an initial force imbalance due to a removal of part of the plasma in the flux rope. Substantial force imbalance also results from insufficient background coronal plasma pressure to offset the magnetic pressure of the flux rope. Due to the nature of the magnetic pressure imbalance we may relate the flux rope's appearance in the corona to the sudden formation of a flux rope in an active region by magnetic reconnection. This scenario may be appropriate since magnetic reconnection in an active region could naturally lead to the sudden formation of an out of equilibrium flux rope in the corona.

\subsection{Insertion of a Flux Rope}

[22] For this simulation, the flux rope is specified by setting $a=0.7, r_{0}=1.0, r_{1}=1.8$ and $a_{1}=0.93$. The flux rope and contained plasma are linearly superimposed upon the existing corona so that the mass and magnetic field of the flux rope are added directly to the corona. The combined system can be seen in Figures 2 and 3. The density of mass contained in the flux rope is further multiplied by a factor of 0.8 , leaving the prominence slightly buoyant. In addition, the equilibrium state of GL requires a significant outward increasing plasma pressure to offset the magnetic pressure in the upper portion of the magnetic flux rope. The background corona is insufficient to provide this pressure and results in a negative pressure when the GL solution is superimposed on the corona. To avoid negative pressure, we limit the depletion of pressure and density in the coronal cavity to 25 percent of the initial coronal values, which leaves the upper portion of the flux rope with unbalanced magnetic pressure. Of the two sources for force imbalance, magnetic pressure is more significant than the filament mass reduction. Removing 20 percent of the filament mass by itself, would only allow the remaining filament to accelerate to a fraction of the Sun's escape velocity.

[23] The introduction of the GL flux rope to the corona has added $5.0 \times 10^{32}$ ergs of magnetic and $3.1 \times 10^{31} \mathrm{ergs}$ of thermal energy as well as $1.0 \times 10^{15}$ gm of plasma to the corona. The added plasma is concentrated in a prominence-type core at the base of the flux rope that is approximately 100 times denser than the ambient corona. The magnetic field strength of the flux rope has a maximum value of approximately 10 Gauss, which is reasonable given the size and height of the flux rope in the corona. The flux rope magnetic field is considerably stronger than the ambient coronal field and is the source of energy to drive the CME. There are some distinct differences between the flux rope parameters used here and those of the earlier simulation by Manchester et al. [2004] that only extended to $32 R_{\odot}$. In this case, the flux rope is more than twice as strong and possesses five times as much energy. Here, the flux rope is located $0.2 R_{\odot}$ closer to the Sun, so that more of the flux is tied to the inner boundary.

\section{Simulation of the CME Event}

[24] In this section, we present the results of a 3-D numerical simulation designed to study the evolution of a flux rope expanding from the corona driving a CME and propagating to $1 \mathrm{AU}$ to form a magnetic cloud. The length of the entire CME computation is 107 hours of simulated time, starting with the initiation of the CME at $t=0$. Time steps were initially very small $\left(\approx 10^{-3} \mathrm{sec}\right)$ because of the low $\beta$ of the flux rope and the associated high Alfvén speed. Time steps exceed $\approx 1 \mathrm{sec}$ when the CME is far from the sun, allowing the simulation to be completed in 129,000 iterations. The calculation required 20,0000 processor-hours and ran some-what faster than real time (76 hours clock time) on 256 processors of the Origin 3800 located at NASA, Ames.

[25] Evolution of the CME begins with rapid acceleration to a speed over $1000 \mathrm{~km} / \mathrm{s}$ in the low corona driving a fast mode MHD shock ahead of the rope. We follow the propagation of the flux rope to $1 \mathrm{AU}$ and find that it decelerates to a speed of $458 \mathrm{~km} / \mathrm{s}$ and continues to drive an extensive shock front. Early evolution of the flux rope is nearly self-similar but magnetic reconnection and interaction with the bi-modal solar wind eventually distort the flux rope. At $1 \mathrm{AU}$, the flux rope has evolved into a well defined magnetic cloud with a maximum field strength of $25 \mathrm{nT}$. Both prolonged southward IMF and shock pressure increase are very geoeffective as is clearly shown in the companion paper that discusses the use of plasma properties of this model at $1 \mathrm{AU}$ to drive a magnetosphere-ionosphere simulation. Extensive use of AMR allows the tracking of small-scale features of the CME to $1 \mathrm{AU}$, such as the shock, and contact discontinuity between the flux rope and the ambient wind. This simulation represents an ongoing effort to develop global space weather models that can track the initiation of CMEs at the solar surface, follow their propagation in interplanetary space, and accurately predict plasma prop- 


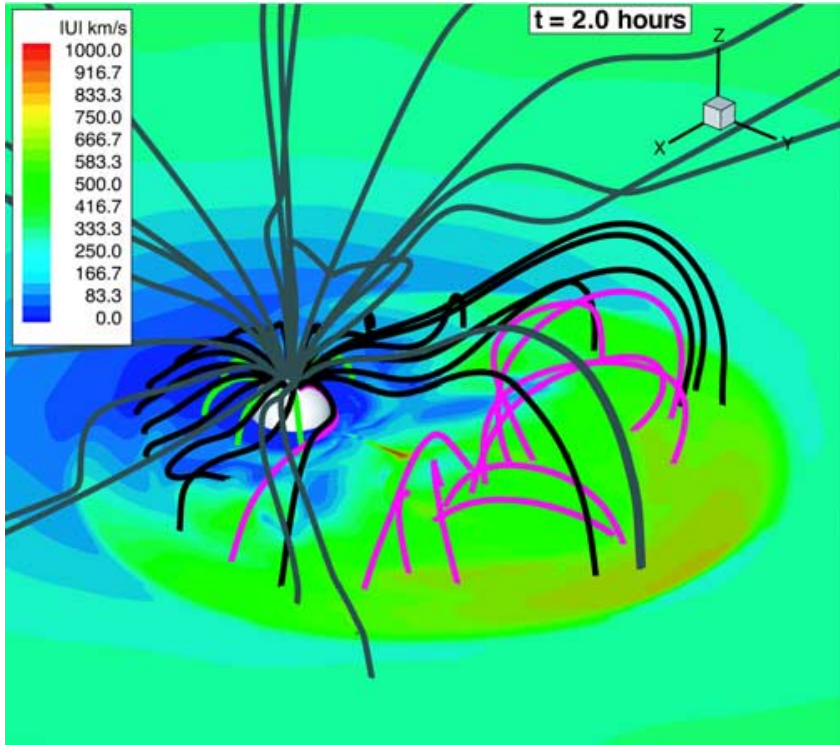

Figure 4. A 3-D representation of the CME is shown two hours after initiation. The color code represents the velocity magnitude in the $x-y$ equatorial plane. Magenta lines show magnetic field lines of the flux rope while black and green lines show magnetic lines of the helmet streamer expanding in front of the flux rope. Gray lines represent the open magnetic flux extending from the pole.

erties at $1 \mathrm{AU}$ that subsequently impact on the Earth's magnetosphere.

\subsection{CME Morphology and Propagation}

[26] Figure 4 presents a 3-D view of the $\mathrm{CME}$ as seen at $t=2.0$ hours from a perspective looking down and between the $x$ and $y$ axis. The false color image shows the velocity magnitude in which a shock front is clearly visible preceding the flux rope. The magnetic field of the flux rope is represented by solid colored magenta lines. The field close to the axis of the flux rope clearly has a strong toroidal component, while the field at the surface of the rope is nearly poloidal. Gray lines show the open field lines of the solar wind, some of which pass through the shock front where the lines bend sharply to wrap around the expanding flux rope. Black and green lines show closed field lines of the streamer belt.

[27] The time evolution of the CME is displayed in Figure 5 with a time series of figures showing the system at $t=8.0,16.0,32.0$ and 64.0 hours. These figures depict the system in 2-D meridional slices ( $y-z$ plane) with false color images of the plasma velocity magnitude upon which solid white lines are superimposed, representing the magnetic field. The flux rope rapidly expands and is expelled from the corona while decelerating. Initial evolution of the flux rope is nearly self similar, but by $t=16$ hours, the front of the rope begins to flatten noticeably. As the flux rope pushes through the dense plasma sheet, radial expansion of the center of the rope stalls, while lateral expansion continues, causing the flux rope to pancake. At high latitude, the extremities of the rope are swept radially forward by the fast solar wind, which bends the sides of the rope when it has traveled more than $50 R_{\odot}$. The combined interaction of the flux rope with the density and velocity structure of the bi-modal solar wind distorts the rope at $1 \mathrm{AU}$ to a crescent shape that is concave outward. A comparison can be made with the 2-D CME model of Riley et al. [2002] in which a flux rope propagates through the heliospheric current sheet without the presence of a bi-modal wind. There, it was found that a dimple formed in the flux rope, but the rope did not bend forward as it does here.

[28] A MHD fast shock front moves ahead of the flux rope, traveling slightly faster than the rope on the $y$ axis while laterally propagating far ahead the rope. We find that the bi-modal nature of the ambient solar wind largely distorts the shock front as it does the flux rope. The wind and magnetosonic speeds are minimal in the heliospheric current sheet and both grow with heliospheric latitude. The shock travels faster at higher latitude in the fast solar wind and with a lower Mach number than at low latitude, resulting in a broad indentation (concave-outward) in the shock front. This same large-scale concave feature of the shock front has been found in earlier work, such as Odstrcil et al. [1996].

[29] A 3-D representation of the distorted flux rope and its interaction with the solar wind is presented in Figure 6. Here, solar wind magnetic field is represented by magenta lines, while the magnetic cloud is shown as an iso-surface of magnetic field strength of $32 \mathrm{nT}$ at $t=60.2$ hours. The field lines show a distorted Parker spiral with field lines bent upward by the fast-mode shock and draping over the magnetic cloud. False color representations of velocity magnitude cover the iso-surface and the equatorial plane, showing the enormous size of the shock front. The numerical grid is shown in black on the equatorial plane where a region of high refinement can be seen extending from the sun to the shock ahead of the CME. The shock and the magnetic cloud structure at $1 \mathrm{AU}$ depend not only on the initial conditions of the corona, but also on the dynamic interaction between the CME and the ambient solar wind. In this case, we find that the flux rope is distorted to a crescent shaped magnetic cloud at $1 \mathrm{AU}$. The shape of solar wind disturbance is important to space weather because it not only determines the magnetic field orientation at the Earth, but also effects the shock inclination, which will be shown to be significant to magnetospheric response in the companion paper.

[30] Figure 7 shows the radial velocity at the front and also at the center of the flux rope plotted as functions of height as solid and dashed lines respectively. Here, we examine the decelerating phase of evolution in which the front experiences an initial drop in velocity from $830 \mathrm{~km} / \mathrm{s}$ to $550 \mathrm{~km} / \mathrm{s}$ by $50 R_{\odot}$. This is followed by a more gradual deceleration that takes the velocity to $430 \mathrm{~km} / \mathrm{s}$ at $1 \mathrm{AU}$. Close to the Sun, the center of the flux rope moves $100 \mathrm{~km} / \mathrm{s}$ slower than the front, indicating a large degree of expansion that lasts for 20 hours until the flux rope reaches approximately $70 R_{\odot}$. The rate of expansion then slowly decreases until $130 R_{\odot}$, at which point, the center of the flux rope begins to overtake the front. This compression is clearly seen in Figure 5d where the flux rope has pancaked, which is most pronounced where the flux rope moves through the dense plasma sheet. Flux rope width at $1 \mathrm{AU}$ is approximately $0.12 \mathrm{AU}$ and including the sheath, extends to more than $0.2 \mathrm{AU}$, which is consistent with the range of magnetic cloud widths found by Gosling et al. [1987]. 

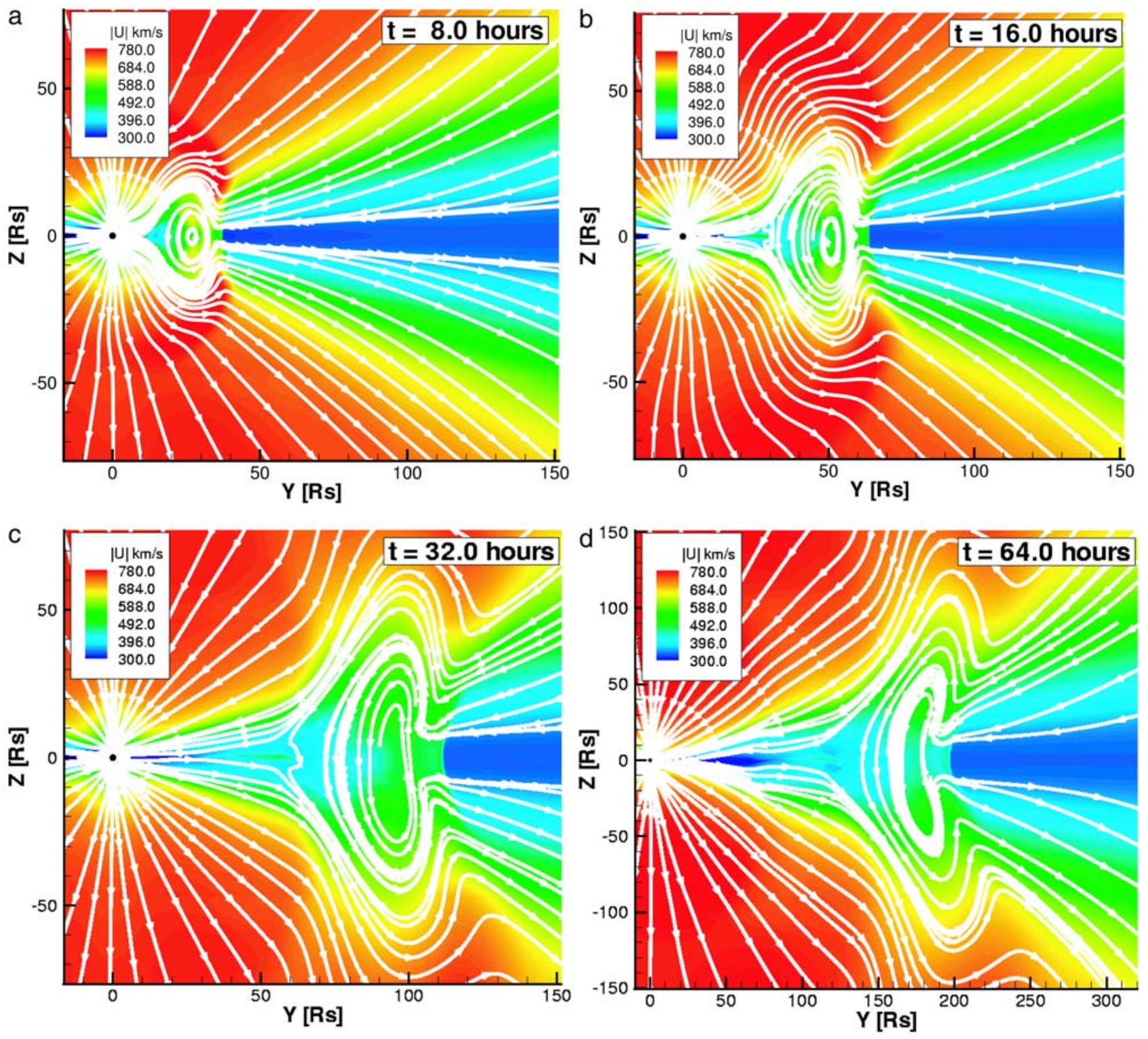

Figure 5. Time evolution of the CME in the $y-z$ meridional plane at (a) $t=8$ hour, (b) $t=16$ hours, (c) $t=32$ hours, (d) $t=64$ hours. Solid white lines display magnetic "streamlines" superimposed upon a false color image of the velocity magnitude.

[31] To further illustrate the expansion and deceleration of the flux rope, we plot height/time curves for the positions on the $y$ axis of the shock, flux rope center, and front in Figure 8 . We also plot the location of the magnetic X-point, marking the point of reconnection between the flux rope and the Sun. At 10 hours, the flux rope front is at $40 R_{\odot}$ and the stand-off distance of the shock is $4.3 R_{\odot}$ The shock front moves steadily ahead of the rope, with the stand-off distance being nearly proportional to the size of the flux rope and hence the distance from the Sun. The shock then reaches $1 \mathrm{AU}$ at $t=69$ hours, $16 R_{\odot}$ ahead of the flux rope, which reaches $1 \mathrm{AU}$ at $t=77$ hours. The shock front extends much farther beyond the rope laterally, reaching the top of the computational domain $z=192 R_{\odot}$ (latitude 57 degrees) by 46 hours, while the flux rope only extends to $z=46 R_{\odot}$ (latitude 19 degrees). At this same time, the shock front extends in the equatorial plane to $x=116 R_{\odot}$ corresponding to an angle of 66 degrees from the $y$ axis. This large-scale shock front traveling through the solar wind (extending to more than 3 times the width of the flux rope) can be clearly seen in Figure 6.

[32] Magnetic reconnection also plays a significant role in the evolution of the CME. At $t=1.0$ hour, the magnetic topology of the system is nearly identical to that of the initial state. As time progresses, a current sheet forms between field lines attaching the flux rope to the coronal boundary. Examination of Figure 5 reveals that reconnection at this current sheet partially severs the flux rope from the boundary and reforms the helmet streamer. The position of the magnetic X-point in Figure 8 shows an interesting dependence on the nature of the flux undergoing reconnection. Flux of the rope reconnects first and as it does so, the distance of the X-point from the Sun increases nearly linearly for 6 hours, at which point the X-point is at $6 R_{\odot}$. 


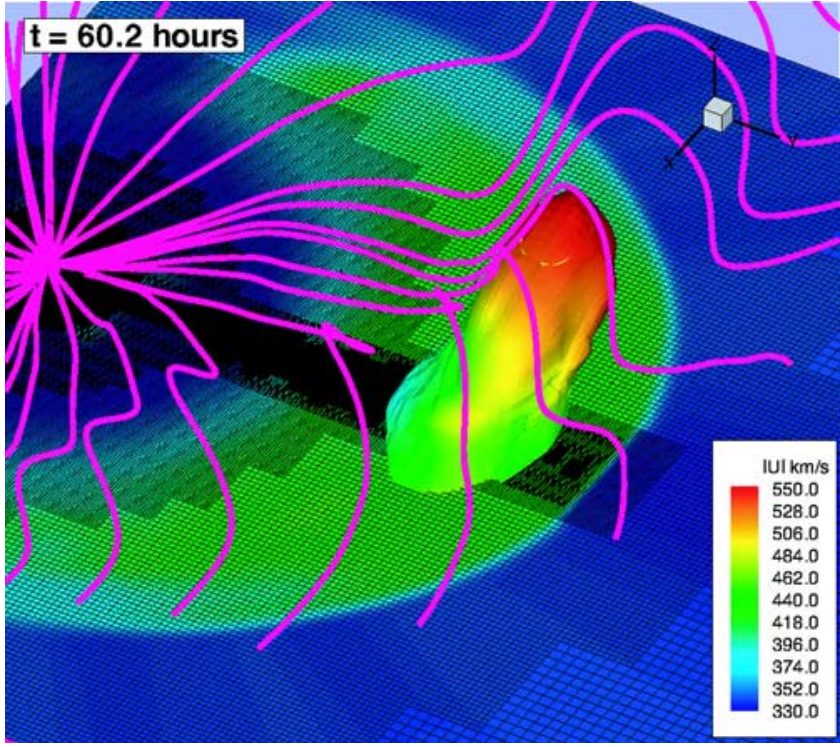

Figure 6. A 3-D representation of the CME shown 60.2 hours after initiation. The color code represents the velocity magnitude in the $x-y$ equatorial plane upon which the computational mesh is superimposed in black. Magenta lines show the spiral magnetic field of the solar wind distorted by the CME-driven shock wave. A 3-D colored iso-surface of magnetic field strength is drawn where $|B|=$ $32 \mathrm{nT}$ to show the magnetic cloud near $1 \mathrm{AU}$.

Next, the flux forming the streamer that enclosed the rope reconnects, and as it does so, the position of the X-point remains nearly stationary at $r \approx 6 R_{\odot}$. Then at $t=20$ hours, the closed flux is exhausted, and the open flux begins to reconnect. Now the wind fills the reconnecting open flux,

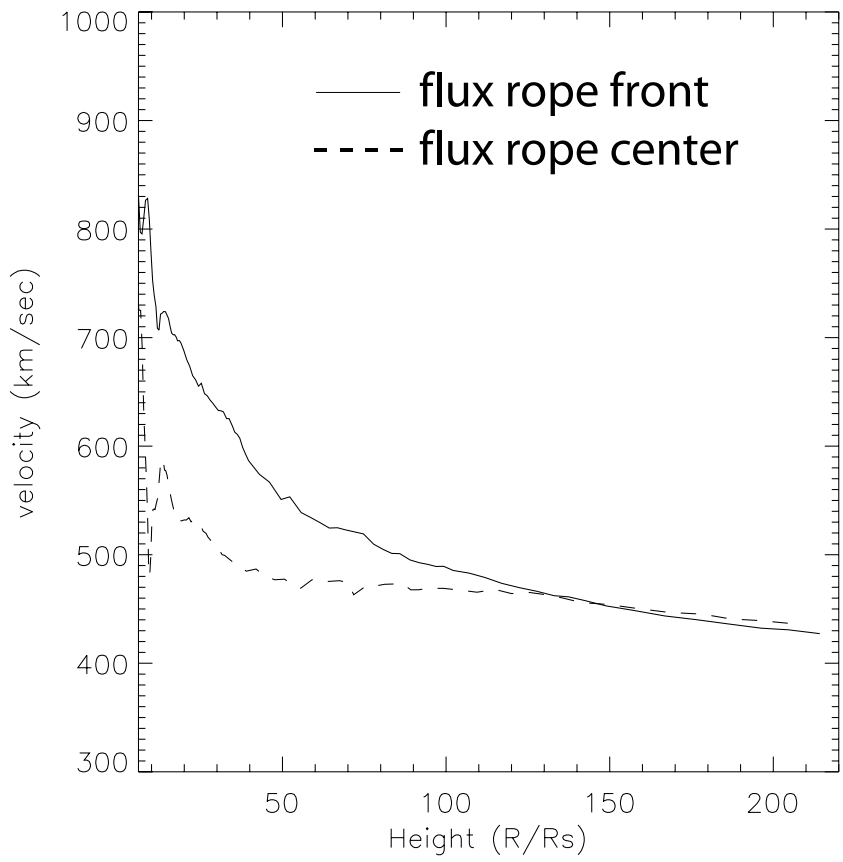

Figure 7. The radial velocity, $u_{r}$, along the $y$ axis, is plotted as a function of height for both the front and center of the flux rope in solid and dashed lines respectively. which expands and reforms the current sheet so that the $\mathrm{X}$-point travels outward at slow solar wind speeds. It is interesting that in our model, the position of the X-point behaves in a way that is distinct to the type of flux reconnecting. This property might prove useful in distinguishing reconnection of open or closed flux in CMEs if similar behavior could be observed. Reconnection in these simulations occurs by way of numerical resistivity, which has been reduced by refining the grid with the use of time dependent AMR.

\subsection{Energetics of the CME}

[33] Since the CME is undriven, the energy for the eruption must come from the pre-event coronal initial state. Given this fact, examining the MHD energy components as a function of time is instructive to determine the exchange of energy during the eruption. The CME erupts with a maximum of $4.2 \times 10^{31}$ ergs of kinetic energy, $8.5 \times 10^{31}$ ergs of thermal energy, and $1.5 \times 10^{30}$ ergs of gravitational energy as shown in Figure 9. The total kinetic + thermal + gravitational energy increase is $1.27 \times 10^{32} \mathrm{ergs}$, which is supplied directly from the magnetic energy of the flux rope, which is liberated as the flux rope expands. Most of the magnetic energy initially goes into thermal energy of the shock heated plasma that is then absorbed by the coronal heat sources. These sources are proportional to the temperature difference between the corona and a target temperature that is greatly exceeded by the shocked plasma. Consequently, the thermal energy exhibits only a short lived increase before falling slightly below the initial value. This heat absorption also dampens the kinetic energy of the CME, which falls shortly after initiation only to slowly

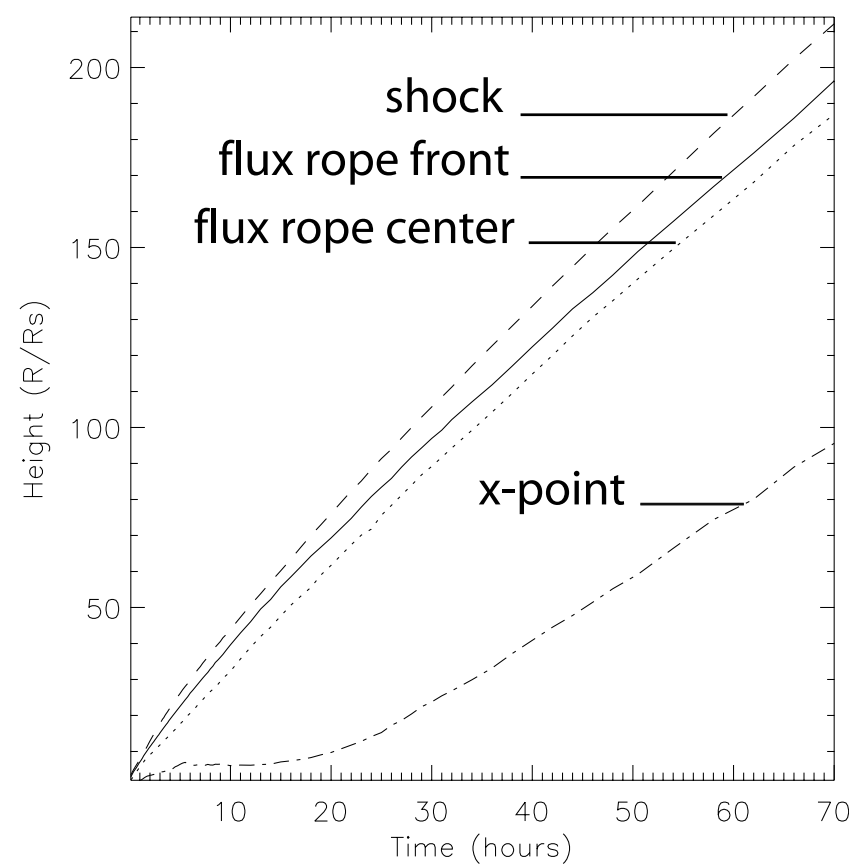

Figure 8. The height, $r$, along the $y$ axis, is plotted as a function of time for the shock front, the front and center of the flux rope as well as the position of the magnetic X-point between the flux rope and the Sun. 


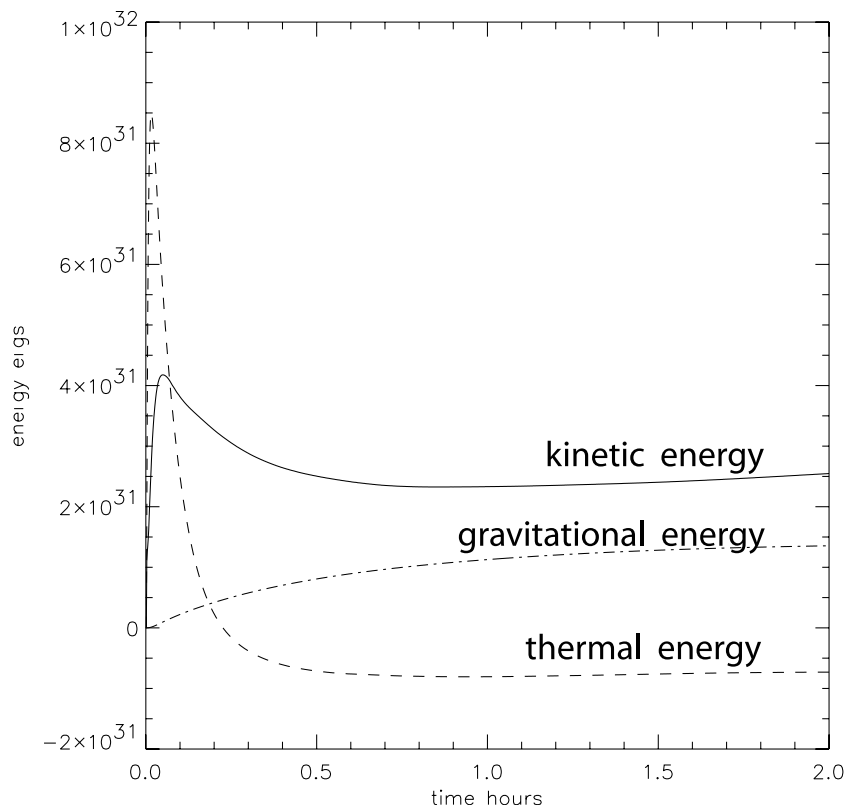

Figure 9. The change in kinetic, thermal and gravitational energies from the initial state are plotted as functions of time as solid, dashed and dot-dashed lines respectively.

increase again after 1 hour. In the CME simulation presented by Manchester et al. [2004], this thermal absorption was prevented. However, without absorption, the reconnection jet that formed behind the flux rope was so strong that it disrupted the flux rope as it traveled further than $50 R_{\odot}$.

\subsection{Properties of the CME at $1 \mathrm{AU}$}

[34] The flux rope evolves to have characteristics at 1 AU commonly associated with magnetic clouds, namely high magnetic field strength, smooth magnetic rotation (north-to-south or south-to-north over a period of approximately a day), low plasma density, and low temperature [Burlaga et al., 1981]. The four panels of Figure 10 show the structure of the magnetic flux rope in a meridional slice $(y-z$ plane) at $t=77$ hours, just when the front of the rope reaches 1 AU. Solid lines are "streamlines" generated using the two components of the magnetic field in the plane of interest while ignoring the component perpendicular to the plane. These lines show the direction of the magnetic field in the plane but do not fully characterize field line topology. False color images show the plasma density, magnetic field strength, velocity magnitude in Figures 10a, 10b, and 10c, respectively, while Figure 10d shows the computational mesh. To make a comparison with in situ measurements, and to provide data for heliospheric-magnetospheric coupling, we also record plasma parameters as a function of time at a point at $1 \mathrm{AU}$ (specifically $x=0 R_{\odot}, y=214 R_{\odot}$ and $z=$ $20 R_{\odot}$ marked by a dot). Quantities at 1 AU plotted as a function of time on the right side of Figure 11 are the radial velocity, plasma number density, temperature, $B z$, and magnetic field magnitude. Data from the Advanced Composition Explorer (ACE) is displayed on the left of Figure 11 (adapted from Lepri et al. [2001]).

[35] Together Figure 10 and Figure 11 reveal much about the CME generated magnetic cloud at 1 AU. Examining the density, in Figure 10a, we find a strong shock that arrives eight hours before the magnetic cloud. As the shock passes the 1 AU point, density increases by a factor of 3.37 from $38 \mathrm{~cm}^{-3}$ to $128 \mathrm{~cm}^{-3}$ and velocity jumps from $370 \mathrm{~km} / \mathrm{s}$ to $458 \mathrm{~km} / \mathrm{s}$. The shock is well resolved with $\frac{1}{8} R_{\odot}$ cells so that the plasma transition across the shock takes only a matter of minutes. Advecting such sharp gradients with an adaptive mesh (see Figure 10d) is essential for propagating geoeffective disturbances to $1 \mathrm{AU}$.

[36] Density peaks behind the shock and then falls slowly until the contact discontinuity at the surface of the flux rope. At this barrier separating flux rope plasma from the shocked solar wind, density falls from $75 \mathrm{~cm}^{-3}$ to $8 \mathrm{~cm}^{-3}$ and remains nearly constant in the cavity of the rope at the $1 \mathrm{AU}$ point. Trailing the flux rope there is a region of rarefaction in the solar wind where the density falls to $1 \mathrm{~cm}^{-3}$. The rarefaction is largely confined to the sheath around the flux rope that contains reconnected open flux previously attached to the Sun. Temperature increases from $5 \times 10^{4} \mathrm{~K}$ to $3 \times 10^{5} \mathrm{~K}$ behind the shock, and then increases to $5 \times 10^{5} \mathrm{~K}$ at the surface of the flux rope. Surface heating of the rope is attributed to magnetic reconnection at the tangential discontinuity separating the rope from solar wind magnetic flux. Increased refinement at this surface would certainly reduce reconnection and plasma heating to more realistic values. Near the center of the rope, we do find that the temperature falls below that of the ambient solar wind.

[37] At the shock, the ambient magnetic field strength increases from $2.6 \mathrm{nT}$ to $7.2 \mathrm{nT}$, largely because of the compression of the toroidal field of the Parker Spiral. The peak field strength at the center of the flux rope at 77 hours is approximately $30 \mathrm{nT}$ (Figure 10b) and reaches a maximum of $25 \mathrm{nT}$ at the chosen magnetosphere location at the $1 \mathrm{AU}$ point. As the flux rope passes the $1 \mathrm{AU}$ point, $B z$, turns northward for four hours, reaching a maximum $20 \mathrm{nT}$. The magnetic field then smoothly rotates southward reaching a similar magnitude, but is more prolonged, lasting greater than 10 hours. The additional southward IMF occurs because open flux of the solar wind drapes around the back of the flux rope, and more importantly, reconnects to generate a sheath of southward IMF trailing the flux rope. The strong northward to southward turning of the IMF and prolonged southward IMF will be shown in the companion paper to produce a strong magnetospheric response.

[38] Making a comparison between the model and ACE data in Figure 11, we find good qualitative agreement in most quantities. The observed shock has a velocity jump of approximately $60 \mathrm{~km} / \mathrm{s}$ and precedes the magnetic cloud by 8 hours, which compares to the $88 \mathrm{~km} / \mathrm{s}$ jump of our model and the same 8 hour shock lead time. We see in the data a clear contact discontinuity in the density profile at the front of the magnetic cloud, reduced density in the cloud, followed by prolonged low density behind the cloud. Our model captures all of these features of the density though our ambient solar wind is more than a factor of two too dense. The flux rope of our model at 1 AU has nearly identical magnetic magnitude as the observed magnetic cloud with a similar fall of in field strength with time. However, our flux rope lasts for only 14 hours compared with the more than 24 hours of the observed cloud. A reason for this may be that our modeled flux rope experienced 

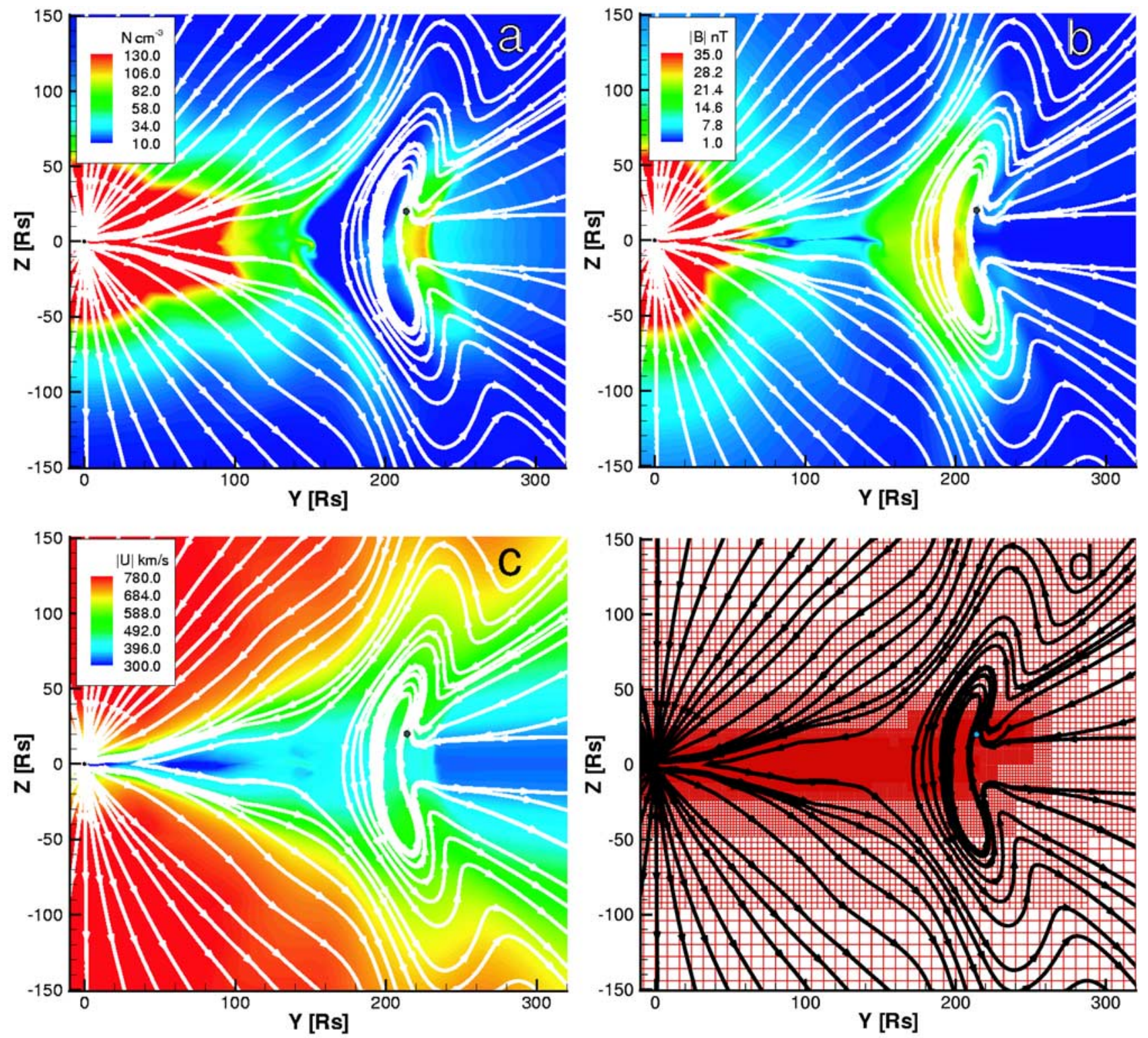

Figure 10. A series of frames shows the CME 77 hours after initiation when the flux rope just reaches 1 AU. All images show the CME in the $y-z$ meridional plane with "streamlines" indicating the direction of the magnetic field in the plane and a dot at $x=0 R_{\odot}, y=214 R_{\odot}$, and $z=20 R_{\odot}$ indicating the position of the point at $1 \mathrm{AU}$ where the time-series data is collected. Frames (a), (b), and (c) depict false color images of the velocity magnitude, plasma density, and magnetic field strength respectively, while frame (d) illustrates the computational mesh. A high degree of refinement is clearly evident for the location that is to be coupled to the Earth's magnetosphere.

considerable compression as it passed through dense plasma near the heliospheric current sheet.

\section{Discussion and Conclusions}

[39] We have investigated the time evolution of a 3-D MHD model of a CME driven by the magnetic pressure and buoyancy of a flux rope in an initial state of force imbalance. The ensuing eruption originates in the low corona and develops as the flux rope and plasma are expelled into the solar wind and propagate to $1 \mathrm{AU}$. Our numerical model possesses many of the observed character- istics of CMEs beginning with the pre-event structure, a dense helmet streamer possessing a cavity and dense core threaded by a ten Gauss magnetic flux rope. In comparison, observations show that the majority of CMEs originate from helmet streamers that overlie quiescent prominences [cf. Hundhausen, 1993] thought to be supported by flux ropes [Low, 2001]. Second, the energy for the eruption comes from the pre-event magnetic configuration and yields $\sim 4 \times 10^{31}$ ergs of kinetic energy that is within the limits commonly observed for CMEs. The CME propagates to 1 AU through a realistic bi-modal steady-state solar wind characteristic of conditions at solar minimum. We find that 

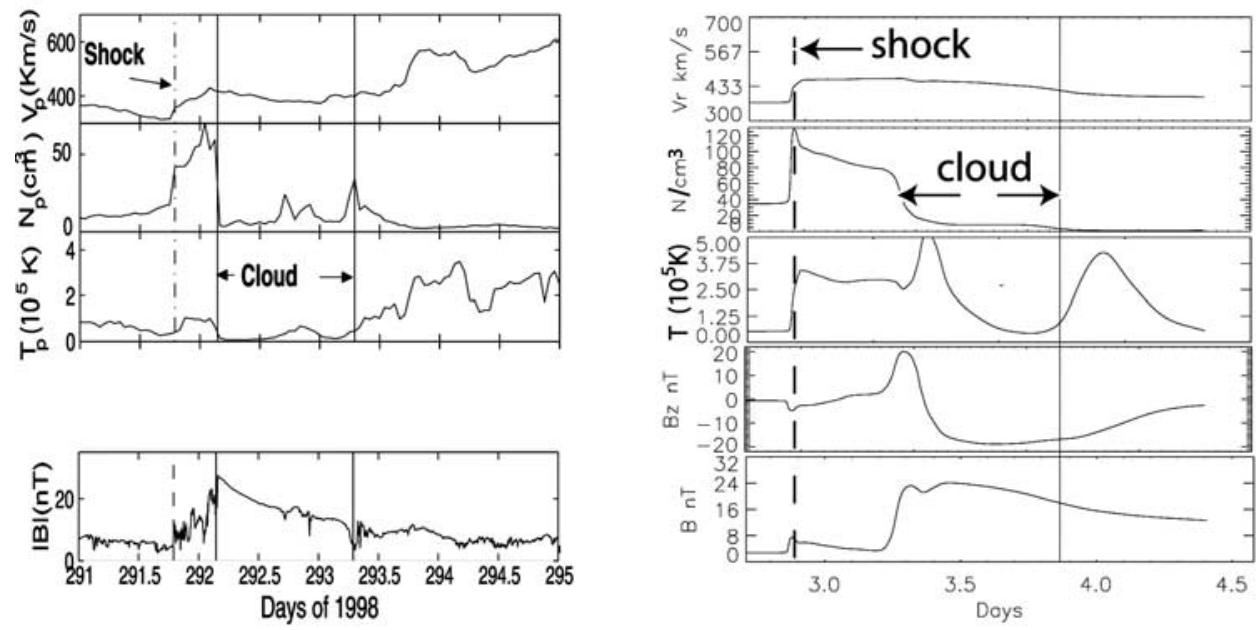

Figure 11. Plasma properties at $1 \mathrm{AU}\left(x=0 R_{\odot}, y=214 R_{\odot}, z=20 R_{\odot}\right)$ for the numerical model are shown as a function of time on the right. From top to bottom, quantities plotted are radial velocity, proton number density, temperature, $B z$ and magnetic field magnitude. On the left, ACE data is plotted for the period October 18-21, 1998 (adapted from Lepri et al. [2001]) showing the same plasma quantities depicted on the right with the exception of $B z$. Note that the time span for the ACE data (4 days) is twice that shown for the modeled event.

on a large scale, both the shock and flux rope are largely distorted by their interaction with this structured wind. At 1 AU the flux rope can be convincingly compared to a magnetic cloud with an enhanced field strength of $25 \mathrm{nT}$, field direction rotation and reduced plasma density.

[40] This simulation represents several significant advances to the previous work of Groth et al. [2000]. First, we have included emerging magnetic flux from the corona in the form of a flux rope that drives the CME. This initiation is more realistic than the density pulse used by Groth et al. [2000] and results in a large IMF in the form of a magnetic cloud at $1 \mathrm{AU}$ that is geoeffective. Furthermore, the speed up in computers in recent years has allowed us to use seven times the number of grid cells as Groth et al. [2000] and along with new AMR refinement criteria, allow a 128 fold increase in numerical resolution at $1 \mathrm{AU}$. The success of our model in capturing many properties of CMEs, including properties of pre-event structures, a bi-modal background solar wind, shock formation and interplanetary propagation of magnetic clouds demonstrates the model's value for studying space weather. With prescribed heating to drive the solar wind, we maintain $\gamma=\frac{5}{3}$ through the entire computational domain. These thermodynamic properties allow us to better capture shocks with appropriate jump conditions compared to simpler models using $\gamma=1.05$ [Linker and Mikić, 1995]. Advanced AMR techniques allow us to resolve geoeffective structures such as shocks and tangential discontinuities to a degree necessary to produce propagation time scales at $1 \mathrm{AU}$ that approach the short dynamic time scale of the Earth's magnetosphere. Future simulations will focus on event studies in which the position of the GL flux rope and its parameters will be chosen to best fit an observed pre-event coronal structure. Also, we will incorporate a global coronal magnetic field based on synoptic magnetograms along with a more realistic, turbulence- driven solar wind that has recently been developed by Roussev et al. [2003].

\section{Appendix A: Mathematical Form of the GL Flux Rope}

[41] The flux rope is obtained by transforming a toroidal magnetic rope contained in a sphere of radius $r_{0}$. The center of the sphere is located at a radial distance of $r_{1}$ on the $y$ axis. The plasma pressure in the flux rope is proportional to the free parameter $a_{1}{ }^{2}$ which also controls the magnetic field strength in the flux rope through pressure balance.

[42] Mathematically, the flux rope magnetic field is written in terms of a scalar function $A$ in spherical coordinates $\left(r^{\prime}, \theta^{\prime}, \phi^{\prime}\right)$ as

$$
\mathbf{F} b=\frac{1}{r^{\prime} \sin \left(\theta^{\prime}\right)}\left(\frac{1}{r^{\prime}} \frac{\partial A}{\partial \theta^{\prime}} \hat{\mathbf{r}}^{\prime}-\frac{\partial A}{\partial r^{\prime}} \hat{\theta}^{\prime}+\alpha_{0} A \hat{\phi}^{\prime}\right)
$$

where

$$
A=\frac{4 \pi a_{1}}{\alpha_{0}^{2}}\left(\frac{r_{0}^{2}}{g\left(\alpha_{0} r_{0}\right)} g\left(\alpha_{0} r^{\prime}\right)-r^{\prime 2}\right) \sin ^{2}\left(\theta^{\prime}\right)
$$

and

$$
g\left(\alpha_{0} r^{\prime}\right)=\frac{\sin \left(\alpha_{0} r^{\prime}\right)}{\alpha_{0} r^{\prime}}-\cos \left(\alpha_{0} r^{\prime}\right)
$$

The pressure inside the flux rope necessary for equilibrium is $\Pi=a_{1} A$, where $a_{1}$ is a free parameter that determines the magnetic field strength and plasma pressure in the flux rope. Here, $r_{0}$ is the diameter of the spherical ball of flux and $\alpha_{0}$ is related to $r_{0}$ by $\alpha_{0} r_{0}=5.763459$ (this number is the smallest 
eigenvalue of the spherical Bessel function, $J_{5 / 2}$ ). The coordinate $\left(r^{\prime}, \theta^{\prime}, \pi^{\prime}\right)$ is centered relative to the heliospheric coordinate system on the $y$ axis at $y=r_{1}$ and oriented such that the circular axis of the flux rope is in the heliospheric equatorial plane.

[43] In the next step, this axisymmetric flux rope is subjected to the mathematical transformation $r \rightarrow r-a$ (where $a$ is the stretching length in the radial direction) that draws space toward the heliospheric origin and distorts the sphere containing the rope to a tear-drop shape. Following this transformation, the magnetic field takes the form:

$$
\begin{gathered}
B_{r}(r, \theta, \phi)=\left(\frac{\Lambda}{r}\right)^{2} b_{r}(\Lambda, \theta, \phi), \\
B_{\theta}(r, \theta, \phi)=\left(\frac{\Lambda}{r} \frac{d \Lambda}{d r}\right) b_{\theta}(\Lambda, \theta, \phi), \\
B_{\phi}(r, \theta, \phi)=\left(\frac{\Lambda}{r} \frac{d \Lambda}{d r}\right) b_{\phi}(\Lambda, \theta, \phi)
\end{gathered}
$$

where $\Lambda=r+a$ and $(r, \theta, \phi)$ are the heliospheric spherical coordinates. Equilibrium within this transformed state demands that the plasma pressure be of the form

$$
p=\left(\frac{\Lambda}{r}\right)^{2}\left(1-\left(\frac{\Lambda}{r}\right)^{2}\right)\left(\frac{b_{r}^{2}}{8 \pi}\right)+\left(\frac{\Lambda}{r}\right)^{2} \Pi
$$

and that density be of the form

$$
\begin{aligned}
\rho= & \frac{1}{F(r)}\left[-\left(\frac{\Lambda}{r}\right)^{2}\left(1-\left(\frac{\Lambda}{r}\right)^{2}\right) \frac{d}{d \Lambda}\left(\Pi+\frac{b^{2}}{8 \pi}\right)\right. \\
& +2 \Pi \frac{\Lambda a}{r^{3}}+\frac{\Lambda a}{4 \pi r^{3}}\left(1-2\left(\frac{\Lambda}{r}\right)^{2}\right) b_{r}^{2} \\
& \left.+\left(\frac{\Lambda}{r}\right)^{2}\left(\frac{a^{2}}{r_{2}}+\frac{2 a}{r}\right)\left(\frac{b_{\theta}^{2}+b_{\phi}^{2}}{4 \pi \Lambda}\right)\right]
\end{aligned}
$$

where $F(r)=G M / r^{2}, G$ being the gravitational constant and $M$ the solar mass.

[44] Acknowledgments. We thank BC Low, Sarah Gibson and Thomas Zurbuchen for discussion concerning this research and S. Lepri for providing a figure depicting plasma data at $1 \mathrm{AU}$. The simulations reported here were carried out on an Origin 3800 supercomputer at NASA AMES. The research for this manuscript was supported by DoD MURI grant F49620-01-1-0359, NSF KDI grant NSF ATM-9980078, NSF CISE grant ACI-9876943, and NASA AISRP grant NAG5-9406 at the University of Michigan. G. Tóth is partially supported by the Education Ministry of Hungary (grant No. FKFP-0242-2000).

[45] Shadia Rifai Habbal thanks Sarah Gibson and Arcadi V. Usmanov for their assistance in evaluating this paper.

\section{References}

Antiochos, S. K., C. R. DeVore, and J. A. Klimchuk (1999), A model for solar coronal mass ejections, Astrophys. J., 510, 485-493.

Axford, W. I., and J. F. McKenzie (1996), The acceleration of the solar wind, in Solar Wind Eight, edited by D. Winterhalter et al., AIP Conf. Proc. Ser., 382, 72-75.

Burlaga, L. E., E. Sittler, F. Mariani, and R. Schwenn (1981), Magnetic loop behind and interplanetary shock: Voyager, Helios, and IMP 8 observations, J. Geophys. Res., 86, 6673.
Burlaga, L. E., L. W. Kein, N. R. Sheeley Jr., D. J. Michels, R. A. Howard, M. J. Koomen, R. Schwenn, and H. Rosenbauer (1982), A magnetic cloud and a coronal mass ejection, Geophys. Res. Lett., 9, 1317.

Chen, J. (1996), Theory of prominence eruption and propagation: Interplanetary consequences, J. Geophys. Res., 101, 27,499.

Chen, J., and D. A. Garren (1993), Interplanetary magnetic clouds: Topology and driving mechanism, Geophys. Res. Lett., 20, 2319-2322.

Chen, P. F., and K. Shibata (2000), An emerging flux trigger mechanism for coronal mass ejections, Astrophys. J., 545, 524-531.

Choe, G. S., and L. C. Lee (1996), Evolution of solar magnetic arcades. II. Effects of resistivity and solar eruptive processes, Astrophys. J., 472, $372-388$.

Dungey, J. W. (1961), Interplanetary magnetic field and the auroral zones, Phys. Rev. Lett., 6, 47.

Fisk, L. A., N. A. Schwadron, and T. H. Zurbuchen (1999), Acceleration of the fast solar wind by the emergence of new magnetic flux, J. Geophys. Res., 104, 19,765-19,772.

Forbes, T. G., and E. R. Priest (1995), Photospheric magnetic field evolution and eruptive flares, Astrophys. J., 446, 377-389.

Gibson, S., and B. C. Low (1998), A time-dependent three-dimensional magnetohydrodynamic model of the coronal mass ejection, Astrophys. J., 493, 460-473.

Gosling, J. T. (1993), The solar flare myth, J. Geophys. Res., 98, 18,937.

Gosling, J. T., D. N. Baker, S. J. Bame, W. C. Feldman, R. D. Zwickl, and E. J. Smith (1987), Bidirectional solar wind electron heat flux events, J. Geophys. Res., 92, 8519.

Groth, C. P. T., D. L. DeZeeuw, T. I. Gombosi, and K. G. Powell (2000), Global three-dimensional MHD simulation of a space weather event: CME formation, interplanetary propagation, and interaction with the magnetosphere, J. Geophys. Res., 105, 25,053-25,078.

Hollweg, J. V., S. Jackson, and D. Galloway (1982), Alfvén waves in the solar atmosphere. III Nonlinear waves on open flux tubes, Sol. Phys., 75, $35-61$.

Hundhausen, A. J. (1987), The origin and propagation of coronal mass ejections, in Solar Wind Six, edited by V. J. Pizzo, T. E. Holzer, and D. G. Sime, Tech. Note NCAR/TN-306+Proc, pp. 181-214, Natl. Cent. for Atmos. Res., Boulder, Colo.

Hundhausen, A. J. (1993), Sizes and locations of coronal mass ejections: SMM observations from 1980 and 1984-1989, J. Geophys Res., 98, $13,177-13,200$

Kumar, A., and D. M. Rust (1996), Interplanetary magnetic clouds, helicity conservation, and current-core flux ropes, J. Geophys. Res., 101, $15,667-15,684$.

Lin, J., and T. G. Forbes (2000), Effects of reconnection on the coronal mass ejection process, J. Geophys. Res., 105, 2375-2392.

Linker, J. A., and Z. Mikić (1995), Disruption of a helmet streamer by photospheric shear, Astrophys. J. Lett., 438, L45-L48.

Lepri, S. T., T. H. Zurbuchen, L. A. Fisk, I. G. Richardson, H. V. Cane, and G. Gloeckler (2001), Iron charge distribution as an indentifier of interplanetary coronal mass ejections, J. Geophys. Res., 106, 29,231-29,238.

Low, B. C. (1983), Explosion of magnetized plasmas from coronae, in Solar and Stellar Magnetic Fields: Origins and Coronal Effects, pp. 467-471, D. Reidel, Norwell, Mass.

Low, B. C. (2001), Coronal mass ejections, magnetic flux ropes, and solar magnetism, J. Geophys. Res., 106, 25,141-25,163.

Manchester, W. B., IV, T. I. Gombosi, I. Roussev, D. L. De Zeeuw, I. V. Sokolov, K. G. Powell, G. Tóth, and M. Opher (2004), Three-dimensional MHD simulation of a flux rope driven CME, J. Geophys. Res., 109, A01102, doi:10.1029/2002JA009672.

Odstrcil, D., and V. J. Pizzo (1999), Three-dimensional propagation of coronal mass ejections (CMEs) in a structured solar wind flow: 1 . CME launched within the streamer belt, J. Geophys. Res., 104, $483-492$

Odstrcil, D., M. Dryer, and Z. Smith (1996), Propagation of an interplanetary shock along the heliosphere plasma sheet, J. Geophys. Res., 101, 19,973-19,986.

Odstrcil, D., D. Odstrcil, J. A. Linker, R. Lionello, Z. Mikíc, P. Riley, V. J. Pizzo, and J. G. Luhmann (2002), Merging of coronal and heliospheric numerical two-dimensional MHD models, J. Geophys. Res., 107(A12), 1493, doi:10.1029/2002JA009334.

Parker, E. N. (1963), Interplanetary Dynamical Processes, Wiley-Interscience, New York.

Pneuman, G. W., and R. A. Kopp (1971), Gas-magnetic field interactions in the solar corona, Sol. Phys., 18, 258-270.

Powell, K. G., P. L. Roe, T. J. Linde, T. I. Gombosi, and D. L. DeZeeuw (1999), A solution-adaptive upwind scheme for ideal magnetohydrodynamics, J. Comput. Phys., 154, 284.

Ridley, A. J., D. L. De Zeeuw, T. I. Gombosi, and K. G. Powell (2001), Using steady state MHD results to predict the global state of the magnetosphere-ionosphere system, Geophys. Res., 106, 30,067-30,076. 
Riley, P., J. A. Linker, Z. Mikić, D. Odstrcil, V. J. Pizzo, and D. F. Webb (2002), Evidence of posteruption reconnection associated with coronal mass ejections in the solar wind, Astrophys. J., 578, 972-978.

Roussev, I. I., T. I. Gombosi, I. V. Sokolov, M. Velli, W. Manchester IV, D. L. De Zeeuw, P. Liewer, G. Tóth, and J. Luhmann (2003), A threedimensional model of solar wind incorporating solar magnetogram observations, Astrophys. J. Lett., 595, L57-L61.

Steinolfson, R. S. (1991), Coronal evolution due to shear motion, Astrophys. J., 382, 677.

Usmanov, A. V., and M. Dryer (1995), A global 3-D simulation of interplanetary dynamics in June 1991, Sol. Phys., 159, 347-370.

Usmanov, A. V., M. L. Goldstein, B. P. Besser, and J. M. Fritzer (2000), A global MHD solar wind model with WKB Alfvén waves: comparison with Ulysses data, J. Geophys. Res., 105, 12,675-12,696.

Wang, Y.-M., and N. R. Sheeley Jr. (1994), Global evolution of the interplanetary sector structure, coronal holes, and solar wind streams during 1976-1993: Stackplot displays based on magnetic observations, J. Geophys. Res., 99, 6597-6608.
Wu, S. T., W. P. Guo, D. J. Michels, and L. F. Burlaga (1999), MHD description of the dynamical relationships between a flux rope, streamer, coronal mass ejection, and magnetic cloud: An analysis of the January 1997 Sun-Earth connection event, J. Geophys. Res., 104, 14,78914,801 .

D. L. De Zeeuw, T. I. Gombosi, W. B. Manchester IV, K. G. Powell, A. Ridley, I. Roussev, and I. V. Sokolov, Center for Space Environment Modeling, University of Michigan, 2455 Hayward Street, Ann Arbor, MI 48109, USA. (darrens@umich.edu; tamas@umich.edu; chipm@umich.edu; powell@engin.umich.edu; ridley@umich.edu; iroussev@umich.edu; igorsok@umich.edu)

G. Tóth, Department of Atomic Physics, Eotvos University, Pazmany setany 1/A, Budapest 1117, Hungary. (gtoth@hermes.elte.hu) 